УДК 517.95

\title{
On Rate of Convergence of Tonelli's and Weak Approximation Methods for Loaded Equations
}

\author{
Yuri Ya. Belov* \\ Kirill V.Korshun ${ }^{\dagger}$ \\ Institute of Mathematics and Computer Science \\ Siberian Federal University \\ Svobodny, 79, Krasnoyarsk, 660041 \\ Russia
}

Received 27.06.2017, received in revised form 05.09.2017, accepted 20.02.2018

We consider the Cauchy problem for a loaded partial differential equation arising in coefficient inverse problem. The convergence of Tonelli's and weak approximation methods for this problem is previously proved. In the article we will prove linear rate of convergence of given methods.

Keywords: differential equation, inverse problem, Cauchy problem, Tonelli's method, weak approximation method, convergence.

DOI: $10.17516 / 1997-1397-2018-11-3-286-294$.

\section{Auxiliary symbols and theorems}

We will use the following notation.

$\Omega$ is bounded domain in the $E^{n}$ space. $x=\left(x_{1}, \ldots, x_{n}\right)$ is a point in $E^{n} . \partial \Omega$ is the boundary of $\Omega . Q_{T}$ is the cylindrical domain $(0, T) \times \Omega$.

$C^{k}(\bar{\Omega})\left(C^{k}(\Omega)\right)$ is the set of all $k$ times continuously differentiable functions of $\bar{\Omega}(\Omega)$, having bounded derivatives up to $k$-th order.

$\Pi_{[0, T]}=\left\{(t, x) \mid t \in[0, T], x \in E^{n}\right\}$.

$\Pi_{[0, T]}^{M}=\left\{(t, x)\left|t \in[0, T], x \in E^{n},\right| x \mid \leqslant M, M-\right.$ const $\}$.

$C^{k, m}([0, T], \Omega)\left(C^{k, m}\left(\Pi_{[0, T]}\right)\right)$ is the set of all functions of $n+1$ variables $\left(t, x_{1}, \ldots, x_{n}\right)$ in $\Pi_{[0, T]}$, which are $k$ times continuously differentiable with respect to $t$ and $m$ times continuously differentiable with respect to spartial variables. All the derivatives listed above are bounded in $\Pi_{[0, T]}$.

$\tau$ is a real-valued parameter, $\tau \in\left[0, \tau_{0}\right], \tau_{0}>0$.

$A, B, C_{i}, i \in \mathbb{N}$, are nonnegative constants depending on the initial data of problems being investigated but do not depending on $\tau$.

Mean value theorem for Definite integral. Let $f(x)$ be a continuous function defined for $x \in[a, b]$. Then

$$
\int_{a}^{b} f(x) d x=f(\xi)(b-a), \quad a \leqslant \xi \leqslant b .
$$

*ybelov@sfu-kras.ru

$\dagger$ tkkorshun@sfu-kras.ru

(C) Siberian Federal University. All rights reserved 


\section{The Cauchy problem}

We consider the inverse problem with unknown coefficient $\mu(t)$ :

$$
\begin{gathered}
\frac{\partial u}{\partial t}=\frac{\partial^{2} u}{\partial x^{2}}+\mu(t) f(t, x), \\
u(0, x)=u_{0}(x) \\
u(t, 0)=\varphi(t)
\end{gathered}
$$

for $(t, x) \in \Pi_{[0, T]}, n=1$.

We consider

$$
u_{0}(0)=\varphi(0),
$$

$u_{0}(x)$ and $f(t, x)$ are bounded and arbitrary smooth functions. By substitution $x=0$ into (1) and using (3) we find unknown $\mu(t)$

$$
\mu(t)=\frac{\varphi^{\prime}(t)-u_{x x}(t, 0)}{f(t, 0)} .
$$

Using (5) we reduce inverse problem (1)-(3) to the Cauchy problem for a loaded equation

$$
\begin{gathered}
\frac{\partial u}{\partial t}=\frac{\partial^{2} u}{\partial x^{2}}+\phi(t, x)\left(\varphi^{\prime}(t)-u_{x x}(t, 0)\right), \\
u(0, x)=u_{0}(x),
\end{gathered}
$$

where $\phi(t, x)=f(t, x) / f(t, 0)$.

\section{Lemma 1}

Consider the one-dimensional problem

$$
\begin{gathered}
\frac{\partial z^{\tau}}{\partial t}=\frac{\partial^{2} z^{\tau}}{\partial x^{2}}-\phi(t, x) z_{x x}^{\tau}(t, 0)+F_{\tau}(t, x), \quad \tau \in\left[0, \tau_{0}\right], \\
\left.z^{\tau}(t, x)\right|_{-\tau \leqslant t \leqslant 0}=0
\end{gathered}
$$

in domain $\Pi_{[0, T]}$. If $z^{\tau}(t, x) \in C^{1,4}\left(\Pi_{[0, T]}\right)$ is a solution to (8), (9), $\phi(t, x) \in C^{0,2}\left(\Pi_{[0, T]}\right)$, $F_{\tau}(t, x) \in C^{0,2}\left(\Pi_{[0, T]}\right),\left|\frac{\partial^{k}}{\partial x^{k}} F_{\tau}(t, x)\right| \leqslant A \tau, 0 \leqslant k \leqslant 2$, then $\left|z^{\tau}(t, x)\right| \leqslant B \tau$, where A, B are constants not depending on $\tau$.

Proof. Note that (8), (9) have a unique solution [2, p. 65]. By applying the maximum principle $\left[3\right.$, p. 16] to $(8),(9)$ in domain $\Pi_{[0, t]}, 0<t \leqslant T$,

$$
\left|z^{\tau}(\eta, x)\right| \leqslant \sup _{E^{1}}\left|z^{\tau}(0, x)\right|+\eta\left(C_{1} \sup _{\Pi_{[0, t]}}\left|z_{x x}^{\tau}(\theta, x)\right|+\sup _{\Pi_{[0, t]}}\left|F_{\tau}(\theta, x)\right|\right), \eta \in[0, t] .
$$

Here, $C_{1}$ is a constant limiting $|\phi(t, x)|$. By applying $\sup _{\Pi_{[0, t]}}$ to both sides of previous inequality,

$$
\sup _{\Pi_{[0, t]}}\left|z^{\tau}(\eta, x)\right| \leqslant \sup _{E^{1}}\left|z^{\tau}(0, x)\right|+C_{2} t\left(\sup _{\Pi_{[0, t]}}\left|z_{x x}^{\tau}(\theta, x)\right|+\sup _{\Pi_{[0, t]}}\left|F_{\tau}(\theta, x)\right|\right), C_{2}=\max \left(C_{1}, 1\right) .
$$


We differentiate (8), (9) two times with respect to $x$ and apply the maximum principle to the resulting equation,

$$
\sup _{\Pi_{[0, t]}}\left|z_{x x}^{\tau}(\eta, x)\right| \leqslant \sup _{E^{1}}\left|z_{x x}^{\tau}(0, x)\right|+C_{3} t\left(\sup _{\Pi_{[0, t]}}\left|z_{x x}^{\tau}(\theta, x)\right|+\sup _{\Pi_{[0, t]}}\left|\frac{\partial^{2}}{\partial x^{2}} F_{\tau}(\theta, x)\right|\right),
$$

where $C_{3}=\sup _{\Pi_{[0, T]}}\left|\phi_{x x}(t, x)\right| . \quad$ We denote $\gamma(t)=\sup _{\Pi_{[0, t]}}\left|z^{\tau}(\theta, x)\right|+\sup _{\Pi_{[0, t]}}\left|z_{x x}^{\tau}(\theta, x)\right|$ $[2$, p. 65]. Note, that

$$
\gamma(0)=\sup _{E^{1}}\left|z^{\tau}(0, x)\right|+\sup _{E^{1}}\left|z_{x x}^{\tau}(0, x)\right|=0 .
$$

By summing up (10) and (11),

$$
\begin{gathered}
\gamma(t) \leqslant \gamma(0)+C_{4} t \sup _{\Pi_{[0, t]}}\left|z_{x x}^{\tau}(\theta, x)\right|+C_{5} \tau t \leqslant C_{6} t \gamma(t)+C_{6} \tau t, \\
C_{4}=C_{2}+C_{3}, \quad C_{5}=2 C_{4} A, \quad C_{6}=\max \left(C_{4}, C_{5}\right) . \\
\left(1-C_{6} t\right) \gamma(t) \leqslant C_{6} \tau t .
\end{gathered}
$$

Let $t^{*}>0$ be a constant such as $1-C_{6} t^{*}=\delta>0, t^{*} K \geqslant T, K$ is integer. Note that $t^{*}$ and $\delta$ not depend on $\tau$. Thus,

$$
\gamma(t) \leqslant \delta^{-1} C_{6} \tau t^{*}, \quad 0 \leqslant t \leqslant t^{*} .
$$

We consider (8), (9) in domain $\Pi_{\left[t^{*}, 2 t^{*}\right]}$. From (13)

$$
\sup _{E^{1}}\left|z^{\tau}\left(t^{*}, x\right)\right|+\sup _{E^{1}}\left|z_{x x}^{\tau}\left(t^{*}, x\right)\right| \leqslant \delta^{-1} C_{6} \tau t^{*} .
$$

Similarly to (10), (11), we prove

$$
\begin{aligned}
& \sup _{\Pi_{\left[t^{*}, t\right]}}\left|z^{\tau}(\eta, x)\right| \leqslant \sup _{E^{1}}\left|z^{\tau}\left(t^{*}, x\right)\right|+C_{2}\left(t-t^{*}\right)\left(\sup _{\Pi_{\left[t^{*}, t\right]}}\left|z_{x x}^{\tau}(\theta, x)\right|+\sup _{\Pi_{\left[t^{*}, t\right]}}\left|F_{\tau}(\theta, x)\right|\right), \\
& \sup _{\Pi_{\left[t^{*}, t\right]}}\left|z_{x x}^{\tau}(\eta, x)\right| \leqslant \sup _{E^{1}}\left|z_{x x}^{\tau}\left(t^{*}, x\right)\right|+C_{3}\left(t-t^{*}\right)\left(\sup _{\Pi_{\left[t^{*}, t\right]}}\left|z_{x x}^{\tau}(\theta, x)\right|+\sup _{\Pi_{\left[t^{*}, t\right]}}\left|\frac{\partial^{2}}{\partial x^{2}} F_{\tau}(\theta, x)\right|\right), \\
& \left(1-C_{6}\left(t-t^{*}\right)\right) \gamma(t) \leqslant \delta^{-1} C_{6} \tau t^{*}+C_{6} \tau t^{*},
\end{aligned}
$$

and finally,

$$
\gamma(t) \leqslant \delta^{-1}\left(\delta^{-1} C_{6} \tau t^{*}+C_{6} \tau t^{*}\right), \quad 0 \leqslant t \leqslant 2 t^{*} .
$$

By applying exact same reasoning in domain $\Pi_{\left[2 t^{*}, 3 t^{*}\right]}$, we prove

$$
\gamma(t) \leqslant \delta^{-1}\left(\delta^{-1}\left(\delta^{-1} C_{6} \tau t^{*}+C_{6} \tau t^{*}\right)+C_{6} \tau t^{*}\right)=C_{6} \tau t^{*} \sum_{i=1}^{3} \delta^{-i}, \quad 0 \leqslant t \leqslant 3 t^{*} .
$$

Making $K$ steps, we prove

$$
\sup _{\Pi_{[0, T]}}|z(t, x)| \leqslant \gamma(T)=C_{6} \tau t^{*} \sum_{i=1}^{K} \delta^{-i}=B \tau .
$$




\section{Tonelli's method}

Let $\tau \in\left[0, \tau_{0}\right]$ be a constant such that $N \tau=T, N$ is integer. We make time shift by $\tau$ in trace of unknown function:

$$
\begin{gathered}
\frac{\partial u^{\tau}}{\partial t}=\frac{\partial^{2} u^{\tau}}{\partial x^{2}}+\phi(t, x)\left(\varphi^{\prime}(t)-u_{x x}^{\tau}(t-\tau, 0)\right), \\
\left.u^{\tau}(t, x)\right|_{-\tau \leqslant t \leqslant 0}=u_{0}(x) .
\end{gathered}
$$

The method of solving problems like (6), (7) by approximation (14), (15) is called Tonelli's method [1].

We consider initial data of the Cauchy problem $(6),(7)$ to satisfy the following conditions:

$$
u_{0}(x) \in C^{k}\left(E^{1}\right), \quad f(t, x) \in C^{0, k}\left(\Pi_{[0, T]}\right), \quad k \geqslant 6, \quad \varphi(t) \in C^{2}([0, T]), \quad f(t, 0) \geqslant \delta>0 .
$$

By (16), the problem (14), (15) is a Cauchy problem for a heat equation with continuous and bounded coefficients, which have a solution $u^{\tau}$ for any $\tau \in\left[0, \tau_{0}\right]$.

Remark. The problem (14), (15) is a particular case of problem (4.1.8), (4.1.9) [2, p. 60] with $a(t)=1, b(t)=c(t)=\gamma=0$. Under Theorem 4.1.1 [2, p. 64], the solution $u^{\tau}(t, x)$ of problem (14), (15) converges to solution $u(t, x)$ of problem (6), (7) with $\tau \rightarrow 0$.

We prove inequalities (4.1.15) [2, p. 61] for $k$ from 1 to 6 and (4.1.16), (4.1.17) [2, p. 61] for $k$ from 0 to 4 exactly same way as in proof of Theorem 4.1.1. Using Theorem 4.1.1,

$$
\begin{gathered}
\left|\frac{\partial}{\partial t} u^{\tau}(t, x)\right| \leqslant C_{7}, \quad\left|\frac{\partial^{k}}{\partial x^{k}} u^{\tau}(t, x)\right| \leqslant C_{8}, \quad k=0, \ldots, 6 . \\
\left|\frac{\partial^{k}}{\partial x^{k}} u(t, x)\right| \leqslant C_{9}, \quad k=0, \ldots, 4, \quad(t, x) \in P i_{[0, T]} .
\end{gathered}
$$

Here and later, $C_{i}$ are constants (maybe different ones) depending on initial data of the problem (6), (7), but not depending on $\tau$.

We denote $z^{\tau}=u^{\tau}-u$. With substraction (6), (7) from (14), (15), function $z^{\tau}$ is a solution to

$$
\begin{gathered}
\frac{\partial z^{\tau}}{\partial t}=\frac{\partial^{2} z^{\tau}}{\partial x^{2}}+\phi(t, x) \tilde{F}_{\tau}(t) \\
z^{\tau}(0, x)=0
\end{gathered}
$$

where

$$
\tilde{F}_{\tau}(t)= \begin{cases}u_{x x}(t, 0)-u_{0}^{\prime \prime}(0), & t \leqslant \tau \\ u_{x x}(t, 0)-u_{x x}^{\tau}(t-\tau, 0), & t>\tau\end{cases}
$$

We add and substract $u_{x x}^{\tau}(t, 0)$ to $\tilde{F}_{\tau}$ :

$$
\tilde{F}_{\tau}(t)= \begin{cases}{\left[u_{x x}(t, 0)-u_{x x}^{\tau}(t, 0)\right]+\left[u_{x x}^{\tau}(t, 0)-u_{0}^{\prime \prime}(0)\right],} & t \leqslant \tau, \\ {\left[u_{x x}(t, 0)-u_{x x}^{\tau}(t, 0)\right]+\left[u_{x x}^{\tau}(t, 0)-u_{x x}^{\tau}(t-\tau, 0)\right],} & t>\tau .\end{cases}
$$

Thus, function $z$ is a solution to

$$
\frac{\partial z^{\tau}}{\partial t}=\frac{\partial^{2} z^{\tau}}{\partial x^{2}}-\phi(t, x) z_{x x}^{\tau}(t, 0)+\phi(t, x) F_{\tau}(t)
$$




$$
z^{\tau}(0, x)=0
$$

where

$$
F_{\tau}(t)= \begin{cases}u_{x x}^{\tau}(t, 0)-u_{0}^{\prime \prime}(0), & t \leqslant \tau \\ u_{x x}^{\tau}(t, 0)-u_{x x}^{\tau}(t-\tau, 0), & t>\tau .\end{cases}
$$

Let $t \leqslant \tau$. By initial condition (15), $u_{x x}^{\tau}(0,0)=u_{0}^{\prime \prime}(0)$; by (14) and (17), $\left|u_{x x t}^{\tau}\right| \leqslant C_{9}$. Thus,

$$
\left|F_{\tau}(t)\right|=\left|u_{x x}^{\tau}(t, 0)-u_{0}^{\prime \prime}(0)\right|=\left|u_{x x}^{\tau}(0,0)-u_{0}^{\prime \prime}(0)+\int_{0}^{t} u_{x x t}^{\tau}(\theta, 0) d \theta\right| \leqslant C_{9} \tau, \quad 0 \leqslant t \leqslant \tau .
$$

Let $t>\tau$. By mean value theorem,

$$
\left|F_{\tau}(t)\right|=\left|u_{x x}^{\tau}(t, 0)-u_{x x}^{\tau}(t-\tau, 0)\right|=\left|u_{x x t}^{\tau}(\theta, 0)\right| \tau \leqslant C_{9} \tau, \quad \tau<t \leqslant T, \quad t-\tau \leqslant \theta \leqslant t .
$$

Thus, $F_{\tau}(t) \leqslant C_{9} \tau$. Under Lemma 1 ,

$$
\sup _{\Pi_{[0, T]}}\left|u^{\tau}-u\right|=\max _{\Pi_{[0, T]}}\left|z^{\tau}(t, x)\right| \leqslant C_{10} \tau .
$$

Theorem 1. If the conditions (16) are valid, then the Tonelli's method converges at linear rate, i.e. $\max _{\Pi_{[0, T]}}\left|u^{\tau}-u\right| \leqslant C \tau$.

\section{Weak approximation method}

Let $\tau>0$ be a constant such that $N \tau=T, N$ is integer. We make a split (see $[3,5,6]$ ) of the problem (6), (7) to two fractional steps, making time shift by ${ }^{\tau} / 2$ in trace of unknown function:

$$
\begin{array}{lrl}
\frac{\partial u^{\tau}}{\partial t}=2 \frac{\partial^{2} u^{\tau}}{\partial x^{2}}, & t \in\left(n \tau,\left(n+{ }^{1} / 2\right) \tau\right], \\
\frac{\partial u^{\tau}}{\partial t}=2 \phi(t, x)\left[\varphi^{\prime}(t)-u_{x x}\left(t-{ }^{\tau} / 2, x\right)\right], & t \in\left(\left(n+{ }^{1} / 2\right) \tau,(n+1) \tau\right], \\
u^{\tau}(0, x)=u_{0}(x), & n=0,1, \ldots, N-1 .
\end{array}
$$

Remark. The problem (23)-(25) is a particular case of problem (2.2.18) investigated in article [7]. Inequalities (17), (18), and convergence of $u^{\tau}$ to $u$ are proved.

We denote averaging function (see [3, p. 41]) as

$$
u_{c p}^{\tau}(t, x)=\frac{1}{\tau} \int_{t}^{t+\tau} u^{\tau}(\theta, x) d \theta
$$

Note that $u_{c p}^{\tau}(t, x)$ is defined in $\Pi_{[0, T-\tau]}$. By applying mean value theorem to right-hand side of (26) $\left(u^{\tau}(t, x)\right.$ is continuous function of $\left.t\right)$,

$$
\begin{gathered}
u_{c p}^{\tau}(t, x)=\frac{1}{\tau} \int_{t}^{t+\tau} u^{\tau}(\theta, x) d \theta=u^{\tau}(\xi, x), \quad t \leqslant \xi \leqslant t+\tau . \\
\frac{\partial^{2}}{\partial x^{2}} u_{c p}^{\tau}(t, x)=\frac{1}{\tau} \int_{t}^{t+\tau} u_{x x}^{\tau}(\theta, x) d \theta=u_{x x}^{\tau}(\xi, x), \quad t \leqslant \xi \leqslant t+\tau .
\end{gathered}
$$


By (17), $u^{\tau}$ and $u_{x x}^{\tau}$ are satisfying Lipschitz condition with respect to $t$, thus

$$
\begin{gathered}
\left|u_{c p}^{\tau}(t, x)-u^{\tau}(t, x)\right|=\left|u^{\tau}(\xi, x)-u^{\tau}(t, x)\right| \leqslant C_{9}|\xi-t| \leqslant C_{9} \tau, \\
\left|\frac{\partial^{2}}{\partial x^{2}} u_{c p}^{\tau}(t, x)-u_{x x}^{\tau}(t, x)\right|=\left|u_{x x}^{\tau}(\xi, x)-u_{x x}^{\tau}(t, x)\right| \leqslant C_{9}|\xi-t| \leqslant C_{9} \tau .
\end{gathered}
$$

We apply averaging function to the problem (23)-(25):

$$
\begin{gathered}
\frac{\partial u_{c p}^{\tau}}{\partial t}=\frac{\partial^{2} u_{c p}^{\tau}}{\partial x^{2}}+\phi(t, x)\left(\varphi^{\prime}(t)-\frac{\partial^{2} u_{c p}^{\tau}}{\partial x^{2}}(t, 0)\right)+F_{\tau}(t, x),(t, x) \in \Pi_{[0, T-\tau]}, \\
u_{c p}^{\tau}(0, x)=u_{0}(x),
\end{gathered}
$$

where

$$
\begin{gathered}
F_{\tau}(t, x)=\frac{1}{\tau} \int_{t}^{t+\tau}\left\{\alpha_{1, \tau}(\theta) u_{x x}^{\tau}(\theta, x)+\alpha_{2, \tau}(\theta) \phi(\theta, x)\left[\varphi^{\prime}(\theta)-u_{x x}\left(\theta-{ }^{\tau} / 2, x\right)\right]-\right. \\
\left.-\frac{\partial^{2} u_{c p}^{\tau}}{\partial x^{2}}(t, x)-\phi(t, x)\left[\varphi^{\prime}(t)-\frac{\partial^{2} u_{c p}^{\tau}}{\partial x^{2}}(t, 0)\right]\right\} d \theta, \\
\alpha_{1, \tau}(t)=\left\{\begin{array}{ll}
2, & t \in\left(n \tau,\left(n+{ }^{1} / 2\right) \tau\right], \\
0, & t \in\left(\left(n+{ }^{1} / 2\right) \tau,(n+1) \tau\right],
\end{array} \quad \alpha_{2, \tau}(t)= \begin{cases}0, & t \in\left(n \tau,\left(n+{ }^{1} / 2\right) \tau\right], \\
2, & t \in\left(\left(n+{ }^{1} / 2\right) \tau,(n+1) \tau\right] .\end{cases} \right.
\end{gathered}
$$

Note that the term

$$
\Psi(t, x)=\frac{\partial^{2} u_{c p}^{\tau}}{\partial x^{2}}(t, x)+\phi(t, x)\left[\varphi^{\prime}(t)-\frac{\partial^{2} u_{c p}^{\tau}}{\partial x^{2}}(t, 0)\right]
$$

in the integrand of $F_{\tau}(t, x)(30)$ does not depend on $\theta, \int_{t}^{t+\tau} \alpha_{1, \tau}(\theta) d \theta=\int_{t}^{t+\tau} \alpha_{2, \tau}(\theta) d \theta=1$, thus

$$
\int_{t}^{t+\tau} \Psi(t, x) d \theta=\int_{t}^{t+\tau}\left\{\alpha_{1, \tau}(\theta) \frac{\partial^{2} u_{c p}^{\tau}}{\partial x^{2}}(t, x)+\alpha_{2, \tau}(\theta) \phi(t, x)\left[\varphi^{\prime}(t)-\frac{\partial^{2} u_{c p}^{\tau}}{\partial x^{2}}(t, 0)\right]\right\} d \theta .
$$

Interval of integration $[t, t+\tau]$ is split by points $\left\{{ }^{j \tau} / 2\right\}, j=0, \ldots, 2 N-1$ to three parts (or two, if $t$ is exactly $\left.{ }^{j \tau} / 2\right)$. Let $I_{s}, s=1,2$ be subsets of interval $[t, t+\tau]$ such that $\alpha_{s, \tau}(\theta) \neq 0, \theta \in I_{s}$. We rewrite (30) as

$$
\begin{aligned}
F_{\tau}(t, x)= & \frac{2}{\tau} \int_{I_{1}}\left(u_{x x}^{\tau}(\theta, x)-\frac{\partial^{2} u_{c p}^{\tau}}{\partial x^{2}}(t, x)\right) d \theta+ \\
& +\frac{2}{\tau} \int_{I_{2}}\left\{\phi(\theta, x)\left[\varphi^{\prime}(\theta)-u_{x x}^{\tau}(\theta-\tau, 0)\right]-\phi(t, x)\left[\varphi^{\prime}(t)-\frac{\partial^{2} u_{c p}^{\tau}}{\partial x^{2}}(t, 0)\right]\right\} d \theta
\end{aligned}
$$

From (31)

$$
\begin{gathered}
\left|u_{x x}^{\tau}(\theta, x)-\frac{\partial^{2} u_{c p}^{\tau}}{\partial x^{2}}(t, x)\right| \leqslant\left|u_{x x}^{\tau}(\theta, x)-u_{x x}^{\tau}(t, x)\right|+\left|u_{x x}^{\tau}(t, x)-\frac{\partial^{2} u_{c p}^{\tau}}{\partial x^{2}}(t, x)\right| \leqslant \\
\leqslant u_{x x t}^{\tau}(\xi, x)|\theta-t|+C_{9} \tau \leqslant C_{11} \tau, \quad t \leqslant \xi \leqslant \theta \leqslant t+\tau . \\
-291-
\end{gathered}
$$


We estimate the second term in $F_{\tau}(t, x)$ :

$$
\begin{aligned}
\mid \phi(\theta, x)\left[\varphi^{\prime}(\theta)-\right. & \left.u_{x x}^{\tau}(\theta-\tau, 0)\right]-\phi(t, x)\left[\left(\varphi^{\prime}(t)-\frac{\partial^{2} u_{c p}^{\tau}}{\partial x^{2}}(t, 0)\right] \mid \leqslant\right. \\
\leqslant & \left|\phi(\theta, x)\left[\varphi^{\prime}(\theta)-u_{x x}^{\tau}(\theta-\tau, 0)\right]-\phi(\theta, x)\left[\varphi^{\prime}(\theta)-u_{x x}^{\tau}(\theta, 0)\right]\right|+ \\
& +\left|\phi(\theta, x)\left[\varphi^{\prime}(\theta)-u_{x x}^{\tau}(\theta, 0)\right]-\phi(t, x)\left[\varphi^{\prime}(t)-u_{x x}^{\tau}(t, 0)\right]\right|+ \\
& +\left|\phi(t, x)\left[\varphi^{\prime}(t)-u_{x x}^{\tau}(t, 0)\right]-\phi(t, x)\left[\varphi^{\prime}(t)-\frac{\partial^{2} u_{c p}^{\tau}}{\partial x^{2}}(t, 0)\right]\right| .
\end{aligned}
$$

By applying the mean value theorem to $(32)$, considering $(16),(17),(27)$ we prove $\left|F_{\tau}(t, x)\right| \leqslant$ $C_{12} \tau$. By differentiation (31) two times with respect to $x$ and applying same reasoning we prove $\left|\frac{\partial^{2}}{\partial x^{2}} F_{\tau}(t, x)\right| \leqslant C_{13} \tau$.

By substraction (6), (7) from (28), (29) (here $z^{\tau}=u_{c p}^{\tau}-u$ ),

$$
\begin{gathered}
\frac{\partial z^{\tau}}{\partial t}=\frac{\partial^{2} z^{\tau}}{\partial x^{2}}-\phi(t, x) z_{x x}^{\tau}(t, 0)+F_{\tau}(t, x),(t, x) \in \Pi_{[0, T-\tau]}, \\
z^{\tau}(0, x)=0 .
\end{gathered}
$$

Under Lemma 1,

$$
\left|z^{\tau}(t, x)\right| \leqslant C_{13} \tau, \quad(t, x) \in \Pi_{[0, T-\tau]} .
$$

Note that $z^{\tau}(t, x) \in C_{\Pi_{[0, T]}^{0,4}}$ and by $(18)\left|z_{t}^{\tau}(t, x)\right| \leqslant C_{9}$, thus $\left|z^{\tau}(t, x)\right| \leqslant C_{13} \tau$ for $(t, x) \in \Pi_{[0, T]}$. By this, (27), and the triangle inequality,

$$
\left|u^{\tau}-u\right| \leqslant\left|u^{\tau}-u_{c p}^{\tau}\right|+\left|u_{c p}^{\tau}-u\right| \leqslant C_{14} \tau
$$

We finally proved

Theorem 2. If the conditions (16) are valid, then weak approximation method converges at linear rate in domain $\Pi_{[0, T]}$.

\section{Future research}

One can prove the Lemma 1 in multidimensional case, i.e. for problem

$$
\begin{gathered}
\frac{\partial z^{\tau}}{\partial t}=\Delta z^{\tau}(t, x)+B\left(z^{\tau}\right)+F_{\tau}(t, x) \\
z^{\tau}(0, x)=0
\end{gathered}
$$

in domain $\Pi_{[0, T]}=\left\{(t, x) \mid 0 \leqslant t \leqslant T, x \in E^{n}, n>1\right\}$, where $B(z)$ is a linear operator with coefficients of $C^{0,2}\left(\Pi_{[0, T]}\right)$ class, depending on function $z$, its first-order partial derivatives, and traces of partial derivatives up to second order. For example,

$$
B\left(z^{\tau}\right)=z^{\tau}(t, x)+\sin \left(t x_{1} x_{2} \ldots x_{n}\right) z^{\tau}(t, 0)+\sum_{i=1}^{n} z_{x_{i}}^{\tau}(t, 0)+\Delta z^{\tau}(t, 0) .
$$

This will provide a method for proving linear rate of convergence of weak approximation method for a range of inverse problems. 
Example. The inverse problem

$$
\begin{gathered}
u_{t}=\Delta u+\sum_{i=1}^{n} u_{x_{i}}+\mu(t) u+f(t, x),(t, x) \in \Pi_{[0, T]}, \\
u(0, x)=u_{0}(x), \\
u(t, 0)=\phi(t),
\end{gathered}
$$

with unknown $\mu(t)$, reduces to a Cauchy problem for a loaded equation

$$
\begin{gathered}
u_{t}=\Delta u+\sum_{i=1}^{n} u_{x_{i}}+\frac{\phi^{\prime}(t)-\Delta u(t, 0)-\sum_{i=1}^{n} u_{x_{i}}(t, 0)-f(t, 0)}{\phi(t)} u+f(t, x), \\
u(0, x)=u_{0}(x) .
\end{gathered}
$$

Provided $f(t, x) \in C^{0,6}\left(\Pi_{[0, T]}\right), u_{0}(x) \in C^{6}\left(E^{n}\right), \phi(t) \in C^{2}([0, T])$, one can use weak approximation method to above problem, by splitting (35), (36) to fractional steps

$$
\begin{aligned}
u_{t}^{\tau}=\alpha_{1, \tau}(t)\left[\Delta u^{\tau}\right. & \left.+\sum_{i=1}^{n} u_{x_{i}}^{\tau}+f(t, x)\right]+ \\
& +\alpha_{2, \tau}(t)\left[\frac{\phi^{\prime}(t)-\Delta u^{\tau}\left(t-\frac{\tau}{2}, 0\right)-\sum_{i=1}^{n} u_{x_{i}}^{\tau}\left(t-\frac{\tau}{2}, 0\right)-f(t, 0)}{\phi(t)} u^{\tau}\right], \\
& u^{\tau}(0, x)=u_{0}(x),
\end{aligned}
$$

proving boundedness [4] of derivatives of $u^{\tau}$ with respect to $x_{i}$ up to sixth order, proving convergence of $u^{\tau}$ to $u$. As in Section 4, by subtraction of (35), (36) from the split-problem and applying averaging function, one can write down the problem for function $z^{\tau}=u^{\tau}-u$ :

$$
\begin{gathered}
z_{t}^{\tau}=\Delta z^{\tau}+B\left(z^{\tau}\right)+F_{\tau}(t, x), \\
z^{\tau}(0, x)=0,
\end{gathered}
$$

where

$$
\begin{aligned}
B\left(z^{\tau}\right)=\sum_{i=1}^{n} z_{x_{i}}^{\tau}(t, x)+\frac{\phi^{\prime}(t)-f(t, 0)}{\phi(t)} z^{\tau}(t, x)+ & \frac{\Delta u^{\tau}(t, 0)-\sum_{i=1}^{n} u_{x_{i}}^{\tau}(t, 0)}{\phi(t)} z^{\tau}(t, x)- \\
& -\frac{u(t, x)}{\phi(t)} \Delta z^{\tau}(t, 0)-\frac{u(t, x)}{\phi(t)} \sum_{i=1}^{n} z_{x_{i}}^{\tau}(t, 0),
\end{aligned}
$$

and $F(t, x) \in C^{0,2}\left(\Pi_{[0, T]}\right)$ is a function satisfying the conditions of Lemma 1. Thus one can prove linear rate of convergence of weak approximation method in this case.

\section{References}

[1] L.Tonelli, Opere scelte. A cura dell'Unione matematica italiana e col contributo del Consiglio nazionale delle ricerce, Roma, Edizioni Cremonese, 1962.

[2] Yu.Ya.Belov et al., Nonclassical and inverse boundary-value problems, Nauchnye zametki, Krasnoyarsk, 2007 (in Russian). 
[3] Yu.Ya.Belov, S.A.Cantor, Weak appromixation method, Krasnoyarsk, Krasnoyarsk Gos. Univ., 1999 (in Russian).

[4] Yu.Ya.Belov, K.V.Korshun, On some inverse problem for Burgers-type equation, Siberian journal of industrial mathematics, 16(2013), no. 3, 28-40.

[5] N.N.Yanenko, G.V.Demidov, Investigation of a Cauchy problem by weak approximation method, Doklady AN SSSR, 167(1966), no. 6, 1242-1244.

[6] A.A.Samarsky, On convergence of fractional steps method for heat transfer equations, Journal of computational mathematics and mathematical physics, 2(1962), no. 6, 1347-1354.

[7] Yu.Ya.Belov, On Estimates of Solutions of the Split Problems for Some Multidimensional Partial Differential Equations, Journal of Siberian Federal University. Mathematics \& Physics, 2(2009), no. 3, 258-270.

\title{
О скорости сходимости метода Тонелли и метода слабой аппроксимации в задачах Коши для нагруженных уравнений
}

\author{
Юрий Я. Белов \\ Кирилл В. Коршун \\ Институт математики и фундаментальной информатики \\ Сибирский федеральный университет \\ Свободный, 79, Красноярск, 660041 \\ Россия
}

\footnotetext{
Рассматривается задача Коши для нагруженного уравнения в частных производных, возникающая при решении коэффициентных обратных задач. Ранее доказана сходимость метода Тонелли и метода слабой аппроксимации для рассматриваемой задачи. В работе доказывается первый порядок сходимости данных методов.

Ключевые слова: дифференциалъные уравнения, обратная задача, задача Коши, метод Тонелли, метод слабой аппроксимации.
} 